\title{
The Incidence of Hepatocellular Carcinoma after Balloon-Occluded Retrograde Transvenous Obliteration
}

\author{
Keiji Yokoyama, ${ }^{1,2}$ Makoto Irie, ${ }^{1}$ Akira Anan, ${ }^{1}$ Masanori Yokoyama, \\ Naoaki Tsuchiya, ${ }^{1}$ Atsushi Fukunaga, ${ }^{1}$ Kazuhide Takata, ${ }^{1}$ Takashi Tanaka, ${ }^{2}$ \\ Genryu Hirano, ${ }^{2}$ Daisuke Morihara, ${ }^{2}$ Yasuaki Takeyama, ${ }^{1}$ Satoshi Shakado, ${ }^{1,2}$ \\ Tetsuro Sohda, ${ }^{1}$ and Shotaro Sakisaka ${ }^{1,2}$ \\ ${ }^{1}$ Department of Gastroenterology and Medicine, Fukuoka University Faculty of Medicine, Fukuoka 814-0180, Japan \\ ${ }^{2}$ The Division of Advanced Clinical Research for Viral Hepatitis and Liver Cancer, Fukuoka University Faculty of Medicine, \\ Fukuoka 814-0180, Japan \\ ${ }^{3}$ Department of Gastroenterology, Murakami Karindoh Hospital, Fukuoka 819-8585, Japan
}

Correspondence should be addressed to Keiji Yokoyama; yokotin@minf.med.fukuoka-u.ac.jp

Received 7 October 2014; Revised 3 March 2015; Accepted 10 March 2015

Academic Editor: Junyong Park

Copyright (C) 2015 Keiji Yokoyama et al. This is an open access article distributed under the Creative Commons Attribution License, which permits unrestricted use, distribution, and reproduction in any medium, provided the original work is properly cited.

\begin{abstract}
Balloon-occluded retrograde transvenous obliteration (BRTO) is a highly effective therapy for gastric varices with liver cirrhosis. We have investigated the incidence of hepatocellular carcinoma (HCC) after BRTO. We enrolled 71 patients with viral hepatitis in which HCC had not appeared with liver imaging findings at the time of BRTO. The overall survival rate after BRTO was $86.8 \%, 76.1 \%$, and $50.5 \%$ at 1,3 , and 5 years. The occurrence rate of HCC after BRTO was $20.9 \%, 41.1 \%$, and $60.7 \%$ at 1,3 , and 5 years, especially showing a higher occurrence of HCC at one year. Meanwhile, the occurrence rate of HCC after treatment which excluded BRTO for esophagogastric varices in patients was $6.3 \%, 19.2 \%$, and $42.5 \%$ at 1,3 , and 5 years. The log-rank test revealed that the occurrence rate of HCC after treatment was significantly higher in the BRTO group compared with that in the non-BRTO group $(P=0.0447)$. The recurrence rate of $\mathrm{HCC}$ after BRTO was $35.8 \%$ and $80.0 \%$ at 1 and 3 years. The present study demonstrated a high incidence of HCC after BRTO in liver cirrhosis patients with viral hepatitis infection. We have suggested the potential for BRTO to accelerate hepatocarcinogenesis.
\end{abstract}

\section{Introduction}

Balloon-occluded retrograde transvenous obliteration (BRTO) was first reported by Kanagawa et al. in 1996 for the treatment of isolated gastric fundal varices [1]. In gastric fundal varices, the 5-year cumulative incidence of bleeding is $44 \%$ in the natural course, and the 1-year survival rate is $48 \%$ in case of bleeding. There is a high need for prophylactic treatment, and many cases are caused by portal hypertension due to liver cirrhosis [2]. In cirrhosis, portal vein pressure rises due to increases in the amount of blood inflow and portal vascular resistance in the liver, leading to the formation of collateral circulation $[3,4]$. BRTO is a breakthrough interventional radiology (IVR) treatment capable of occluding all inflow channels in one procedure. BRTO is also known to improve hepatic encephalopathy by occluding large shunts and increasing the blood flow in the hepatic portal vein [5]. Currently, BRTO has been established as one of the effective treatments of gastric varices and hepatic encephalopathy [6].

Both hepatitis B virus (HBV) and hepatitis $\mathrm{C}$ virus (HCV) are highly prevalent in Asian countries including Japan where high incidence of hepatocellular carcinoma (HCC) is reported. Since more than $80 \%$ of HCC patients are infected by HBV or HCV in Japan, these two types of hepatitis virus are important factors in hepatocarcinogenesis. In particular, cases with liver cirrhosis constitute a high-risk group. Patients who meet the indication for BRTO have a background of liver 


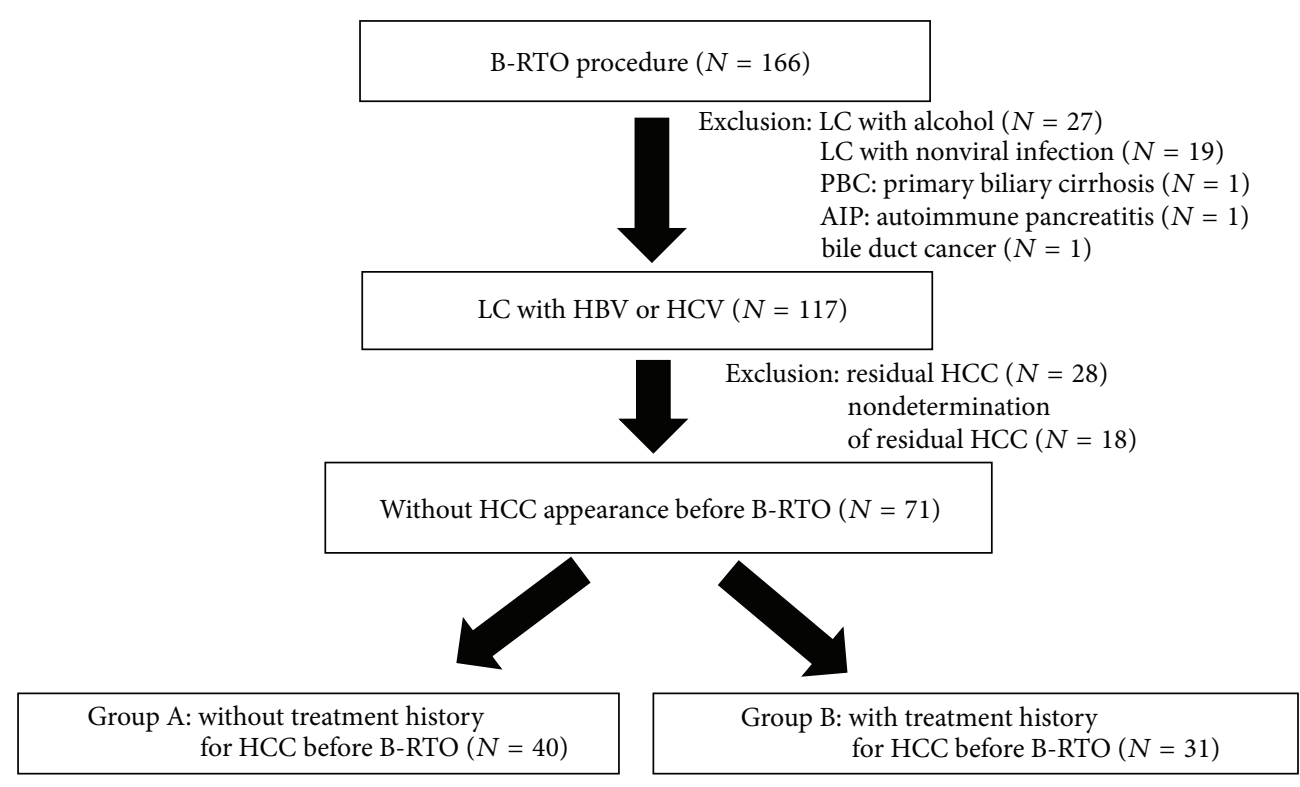

FIgURE 1: The flow chart for all cases divided into two groups.

cirrhosis due to hepatitis virus infection as well as a high risk of HCC. However, very few studies have investigated the incidence of HCC after BRTO.

In the present study, we have investigated the survival and incidence rate of HCC after BRTO in HBV or HCV infected patients by statistical analysis.

\section{Materials and Methods}

2.1. Study Design. From April 1993 to December 2013, BRTO was performed for 166 patients with liver cirrhosis at the Department of Gastroenterology and Medicine, Fukuoka University Faculty of Medicine. 117 out of 166 patients were infected with HBV or HCV. Of the 117 patients, 28 patients, who had coexistent HCC when BRTO was performed, and 18 patients, for whom the presence of residual HCC could not be completely negligible because they had undergone treatment for HCC within 30 days before or after BRTO, were excluded. Seventy-one patients who had no imaging findings of HCC when BRTO was performed were enrolled in this study (Figure 1). Within this number, 66 patients were positive for $\mathrm{HCV}$, and 5 patients were positive for HBV.

The imaging findings were obtained by contrast enhanced computed tomography (CECT) and/or gadolinium-ethoxybenzyl-diethylenetriamine pentaacetic acid-enhanced magnetic resonance imaging (Gd-EOB-DTPA enhanced MRI) performed within 30 days before BRTO. A radiologist and a hepatologist interpreted each result.

Seventy-one patients were divided into two groups: group A, patients without history of HCC before BRTO $(N=40)$ and group $\mathrm{B}$, patients with history of treatment for HCC and diagnosed with no HCC appearance when BRTO was performed $(N=31)$. We investigated the period from each BRTO to treatment for HCC (group A: first-line treatment for HCC occurrence and group B: treatment for HCC recurrence). Patient characteristics for each group are shown in Table 1. FIB-4 (a simple formula for predicting liver fibrosis, in accordance with reporting by Vallet-Pichard), age (years) $\times$ AST [IU/L]/(Plt [109/L] $\times($ ALT [IU/L]) 1/2), is shown [7].

In group B, the treatment modality of HCC in 31 patients before BRTO treatment was as follows: transcatheter arterial chemoembolization (TACE) in 18 cases and percutaneous radiofrequency ablation (RFA) in 13 cases.

We also enrolled as the non-BRTO group: 51 patients with HBV or HCV infection and without history of HCC before treatment which excluded BRTO for esophagogastric varices in the same period. In the non-BRTO group, the treatment modality of esophagogastric varices in 51 patients was as follows: endoscopic variceal ligation (EVL) in 6 cases, endoscopic injection sclerotherapy (EIS) in 13 cases, and combination therapy for EVL and EIS in 32 cases.

Our research protocol was approved by the Ethics Committee of Fukuoka University Hospital. All patients gave written informed consent prior to their participation.

2.2. Follow-Up and Assessment of Disease Progression. All patients were followed up for HCC status at least every three months by diagnostic imaging including abdominal ultrasonography (US), CECT, and Gd-EOB-DTPA enhanced MRI. Determination of HCC incidence after BRTO was set when typical imaging findings were obtained or tumors were histologically confirmed by ultrasound-guided tumor biopsy.

2.3. BRTO Methods. BRTO was performed as follows: first, an ordinary transarterial portography via the splenic or superior mesenteric artery was performed using the 5-French system catheter (Medikit, Tokyo, Japan) via the right transfemoral approach to assess the status of the varices or portosystemic shunts. Then, an 8- to 10-Fr sheath catheter was inserted 
TABLE 1: Comparison of patient characteristics between the two groups: group A, patients without history of HCC before B-RTO $(N=40)$ and group B, patients with history of treatment for HCC and diagnosed with no HCC appearance when B-RTO was performed $(N=31)$.

\begin{tabular}{|c|c|c|c|}
\hline & Group A $(N=40)$ & Group B $(N=31)$ & $P$ value \\
\hline Age, years & $61.5 \pm 1.50$ & $70.1 \pm 1.70$ & $0.0003^{*}$ \\
\hline \multicolumn{4}{|l|}{ Gender } \\
\hline Male & 24 & 13 & \\
\hline Female & 16 & 18 & 0.131 \\
\hline \multicolumn{4}{|l|}{ Etiology } \\
\hline $\mathrm{HCV}$ & 36 & 30 & \\
\hline $\mathrm{HBV}$ & 4 & 1 & 0.269 \\
\hline FIB4 index & $9.19 \pm 1.04$ & $9.69 \pm 1.18$ & 0.751 \\
\hline Child-Pugh score & $7.37 \pm 0.19$ & $7.16 \pm 0.22$ & 0.464 \\
\hline $\log$ AFP & $1.22 \pm 0.13$ & $1.41 \pm 0.13$ & 0.313 \\
\hline $\log \mathrm{DCP}$ & $1.13 \pm 0.07$ & $1.48 \pm 0.07$ & $0.0007^{*}$ \\
\hline Injected 5\% EOI, mL & $27.8 \pm 2.01$ & $23.9 \pm 2.28$ & 0.198 \\
\hline
\end{tabular}

FIB4 index $=$ age $($ years $) \times$ AST $[$ IU/L]/(Plt $[109 / \mathrm{L}] \times($ ALT $[$ IU/L] $) 1 / 2)$.

HCV: hepatitis C virus, HBV: hepatitis B virus, AFP: alpha-fetoprotein, DCP: des-gamma-carboxy prothrombin, and EOI: ethanolamine oleate-iopamidol mixture.

${ }^{*} P$ values less than 0.05 were considered significant.

through the right femoral vein for BRTO. Subsequently, a 6or 8-Fr balloon catheter $(20 \mathrm{~mm}$ or $30 \mathrm{~mm}$ balloon; Clinical Supply, Gifu, Japan) was selectively inserted at the proximal or downstream side of the shunting vessels, through which digital subtraction angiography (DSA) was obtained using slow manual injection of contrast medium (Iopamiron 200, Bayer Schering, Germany) with the balloon inflated. When there was no visualization of the varices or the whole shunts due to the presence of collateral vessels, these collaterals were embolized using microcoils (Diamond, Boston Scientific, Natick, MA) via a 2.9-Fr microcatheter system (FasTracker, Boston Scientific, Natick, MA). Slow manual injection was repeated until the gastric varices or whole shunt vessels were opacified and then a microcatheter was advanced into the center of the varices or the shunt vessel, where a sclerosing agent (5\% ethanolamine oleate-iopamidol mixture: EOI) was slowly injected via a microcatheter with the balloon inflated until it opacified the entire gastric varices or the shunt vessels. Microcoils were sometimes added to enhance the thrombogenic effect of the sclerosing agent. A total of 4000 units of human haptoglobin (Benesis, Osaka, Japan) were intravenously administered before injection of the sclerosing agent to prevent hemolysis and subsequent renal failure, which might be induced by the EOI. The balloon was kept inflated overnight, and the next morning it was deflated after test injection to confirm that the varices or shunt vessels were completely thrombosed (Figure 2). Transarterial portography was repeated to confirm the complete disappearance of the varices or portosystemic shunts, and the procedure was terminated. CECT was performed to assess the status of the varices or portosystemic shunts 4 weeks after BRTO.

2.4. Statistical Analysis. Results are presented as the mean \pm standard error (SE). The methods of comparison between groups included the $t$-test and chi-square test using JMP version 10.0 (SAS Institute, Cary, NC, USA). The cumulative

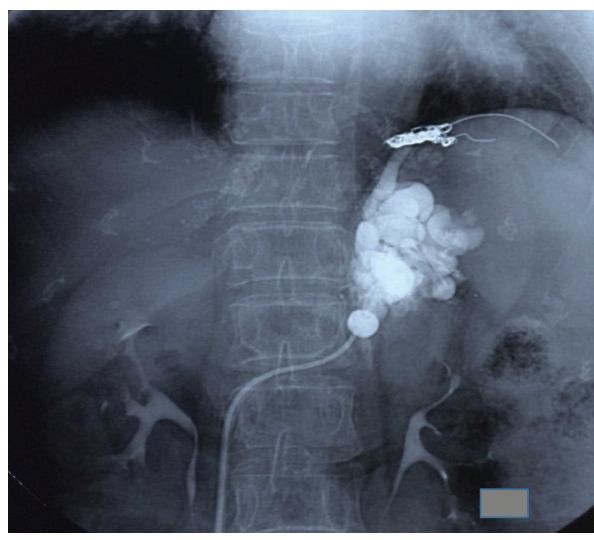

FIGURE 2: Radiograph after embolization of the gastric varices demonstrates complete and adequate accumulation of the 5\% ethanolamine oleate-iopamidol mixture in the varices.

survival curve and the occurrence/recurrence curve of HCC were calculated according to the Kaplan-Meier method. A log-rank test was used for the comparison between each curve. $P$ values less than 0.05 were considered significant.

\section{Results}

At first, in a comparison of patient characteristics between group A and group B, age at treatment was significantly lower in group A $(P=0.0003)$. Furthermore, Log DCP was significantly higher in group $\mathrm{B}(P=0.0007)$ (Table 1$)$.

Secondly, in a comparison of patient characteristics between group A and the non-BRTO group, no statistically significant differences were observed between the two groups with respect to functional hepatic reserve, fibrosis markers, and tumor markers (Table 2). 
TABLE 2: Comparison of patient characteristics between the two groups: group A: patients without history of HCC before B-RTO $(N=40)$ and the non-BRTO group: patients with viral hepatitis and without history of HCC before treatment excluded BRTO for esophagogastric varices $(N=51)$.

\begin{tabular}{lccc}
\hline & Group A $(N=40)$ & Non-BRTO $(N=51)$ & $P$ value \\
\hline Age, years & $61.5 \pm 1.63$ & $60.0 \pm 1.44$ & \\
Gender & 24 & 30 & \\
$\quad$ Male & 16 & & \\
$\quad$ Female & & 41 & 0.480 \\
Etiology & 36 & 10 & \\
HCV & 4 & $7.80 \pm 0.79$ \\
HBV & $9.19 \pm 0.89$ & $6.98 \pm 0.17$ \\
FIB4 index & $7.37 \pm 0.20$ & $1.18 \pm 0.08$ & 0.207 \\
Child-Pugh score & $1.22 \pm 0.11$ & $1.21 \pm 0.03$ & 0.244 \\
log AFP & $1.13 \pm 0.05$ & 0.138 \\
$\log$ DCP & & & 0.759 \\
\hline
\end{tabular}

FIB4 index $=$ age $($ years $) \times$ AST $[$ IU/L] $/($ Plt $[109 / \mathrm{L}] \times($ ALT $[$ IU/L] $) 1 / 2)$.

HCV: hepatitis C virus, HBV: hepatitis B virus, AFP: alpha-fetoprotein, and DCP: des-gamma-carboxy prothrombin.

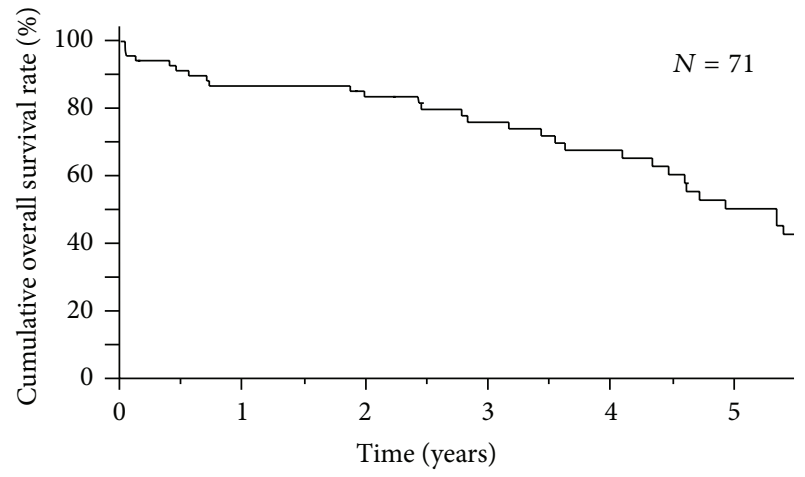

FIGURE 3: The cumulative overall survival rate after B-RTO in 71 patients who were without HCC appearance on imaging findings when B-RTO was performed.

3.1. Survival after BRTO. The cumulative overall survival rate after BRTO was $86.8 \%, 83.6 \%, 76.1 \%$, and $50.5 \%$ at $1,2,3$, and 5 years, respectively (Figure 3 ).

3.2. HCC Status. The cumulative overall incidence rate of HCC after BRTO was $25.4 \%, 44.0 \%, 55.5 \%$, and $64.4 \%$ at 1 , 2,3 , and 5 years, respectively (Figure 4 ).

In group A, the cumulative occurrence rate of $\mathrm{HCC}$ after BRTO in patients without a history of HCC was $20.9 \%$, $27.2 \%, 41.1 \%$, and $60.7 \%$ at $1,2,3$, and 5 years, respectively, and showed a high rate of approximately $20 \%$ at one year (Figure 5).

In group $\mathrm{B}$, the cumulative recurrence rate of $\mathrm{HCC}$ after BRTO in patients with a history of HCC was $35.8 \%, 65.0 \%$, and $80.0 \%$ at 1,2 , and 3 years, respectively, and showed a recurrence in approximately $30 \%$ annually (Figure 6).

As described above, in group B, the treatment modality of HCC before BRTO was divided into the TACE group

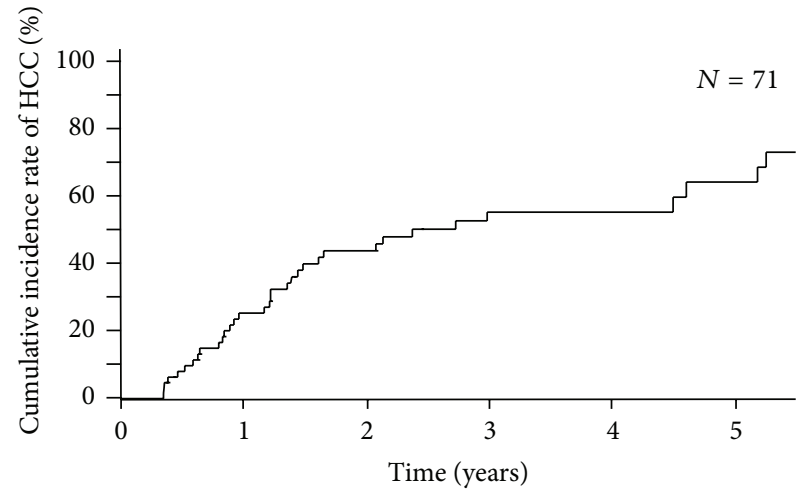

FIGURE 4: The cumulative overall incidence rate of HCC after BRTO in 71 patients who were without HCC appearance on imaging findings when B-RTO was performed.

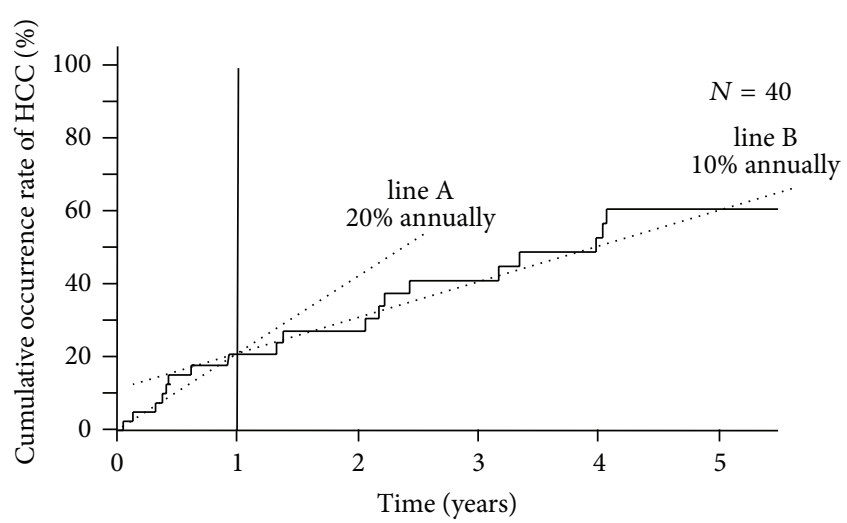

FIgURE 5: The cumulative occurrence rate of HCC after B-RTO in 40 patients without a history of HCC (full line) showed approximately $20 \%$ occurrence within one year after B-RTO (dotted line A), subsequently $10 \%$ annually (dotted line B). 


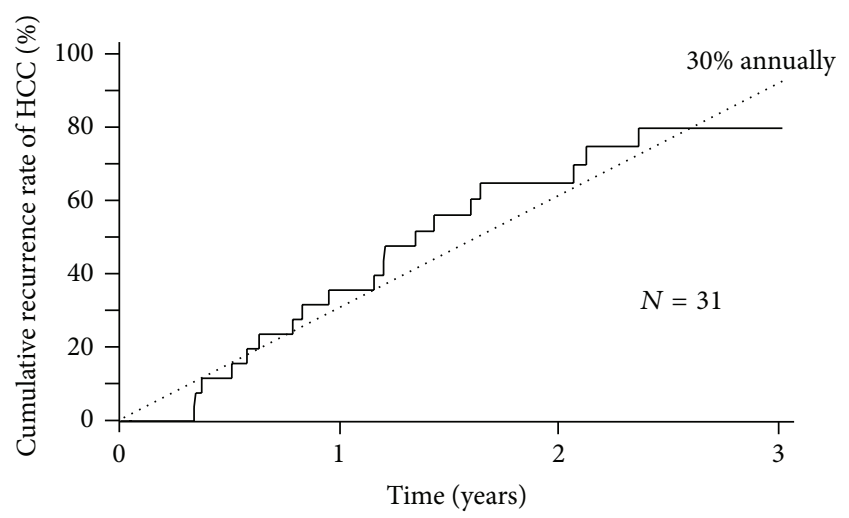

FIGURE 6: The cumulative recurrence rate of HCC after B-RTO in 31 patients with a history of HCC (full line) was approximately $30 \%$ annually (dotted line).

$(N=18)$ and the RFA group $(N=13)$. A comparison between the TACE group and the RFA group showed no statistically significant difference in the recurrence rate of $\operatorname{HCC}(P=0.4768)$.

The cumulative occurrence rate of HCC after non-BRTO treatment for esophagogastric varices in patients was $6.3 \%$, $16.2 \%, 19.2 \%$, and $42.5 \%$ at $1,2,3$, and 5 years, respectively. In a comparison of the cumulative occurrence rate of HCC after treatment between the BRTO group (group A) and the nonBRTO group, the log-rank test revealed that the cumulative occurrence rate of HCC after treatment was significantly higher in the BRTO group, compared with that in the nonBRTO group $(P=0.0447)$ (Figure 7).

\section{Discussion}

The present study revealed that the occurrence of HCC showed a high rate of approximately $20 \%$ in patients without a history of HCC within one year after BRTO. Subsequently, it showed slightly higher rate (approximately $10 \%$ annually). On the other hand, the non-BRTO group was comparable to the common occurrence rate of HCC associated with liver cirrhosis type C ( $8 \%$ annually) [8]. Previous reports have demonstrated that hepatic functional reserve and fibrosis are improved temporarily by increased blood flow through the portal vein following BRTO [9]. It has been speculated that improvement of liver fibrosis following BRTO would potentially inhibit the occurrence of HCC. However, in the present study, the HCC occurrence rate increased immediately after BRTO, which was completely contrary to the previous prediction. Therefore, the results of this study first indicated that BRTO increases the risk of HCC occurrence.

In this study, the recurrence rate of HCC in patients with a history of treatment for HCC before BRTO (group B) was approximately $30 \%$ annually. In the RFA group, the cumulative recurrence rate of HCC after BRTO was $33.3 \%, 68.8 \%$, and $89.9 \%$ at 1,2 , and 3 years, respectively. Recently, Ikeda reported the recurrence rate of HCC after RFA was $11.3 \%, 40.4 \%$, and $53.3 \%$ at 1,2 , and 3 years, respectively, within the indication criteria for percutaneous ablation therapy ( $<3$ nodules, $<3 \mathrm{~cm}$ ), while another study reported $10-25 \%$ and $20-60 \%$ in one-year and three-year recurrence rate $[10,11]$. We have documented that it was $17 \%, 39 \%$, and $43 \%$ at 1,2 , and 3 years, respectively [12]. Therefore, the results of this study suggested the possibility that BRTO accelerated the recurrence of HCC. Meanwhile, in a comparison of patient characteristics between group $\mathrm{A}$ and group B, Log DCP, known as one of tumor markers of HCC, was significantly higher in group B. As a reason for that we also considered there was a possibility that early HCCs which cannot appear by diagnostic imaging already existed.

The seventy-one patients enrolled in this study were positive for HBV or HCV, and, therefore, the liver had a high carcinogenic potential. It has been reported that direct action by either virus and the indirect action of acceleration of carcinogenesis by the sequelae of chronic infection in the host are the carcinogenic mechanisms in HBV or HCV infected patients [13]. Nevertheless, the carcinogenic mechanism has not been completely elucidated and a number of points remain to be clarified. However, this high carcinogenic potential may influence the incidence of HCC after BRTO. On the basis of the results of this study, we formed two hypotheses for HCC occurrence and cancer growth due to BRTO. The mechanisms of the hypotheses are shown in Figure 8.

The first hypothesis is that BRTO leads increased portal blood flow to the liver, resulting in increased insulin delivery to hepatocytes. Insulin is known to be a growth factor for cancer and the doubling time of HCC was significantly shorter in patients with hyperinsulinemia [14]. Moreover, epidemiological studies from various countries have revealed that hyperinsulinemia is strongly implicated in HCC occurrence $[15,16]$. The potential mechanism is as follows: influx of insulin into the liver inhibits the production of insulin-like growth factor binding protein-1 (IGFBP-1) in the liver, which increases the active form insulin-like growth factor-1 (IGF1) [17]. Consequentially, cell proliferation and differentiation are induced and the growth of HCC is accelerated.

The other hypothesis is the possibility that BRTO induces increased interleukin-6 (IL-6) concentration in the liver similar to percutaneous transhepatic portal vein embolization (PTPE). PTPE has been employed as a pretreatment to prevent liver failure after extended hepatectomy in cases with HCC and advanced biliary cancer [18]. PTPE increases portal blood flow as BRTO dose. IL-6 is produced by sinusoidal endothelial cells after PTPE because of continuous expanding stimulation of endothelial cells in the portal vein in the process of regeneration of the nonembolized lobe [19]. IL-6 accelerates neovascularization by inducing vascular endothelial growth factor (VEGF) production and contributes to the regeneration and repair of liver tissue. When IL- 6 concentrations increase in the blood, IL- 6 binds the receptor on the surface of hepatocytes and regulates the expression of VEGF. These functions seem to be involved in the persistence of advancement of HCC [20,21]. Nakagawa et al. reported that morbidity of HCC tended to be higher as serum IL-6 levels increased in 330 patients with chronic hepatitis C [22]. Because BRTO increases blood flow in the entire liver, as in the nonembolized liver lobe after PTPE, 


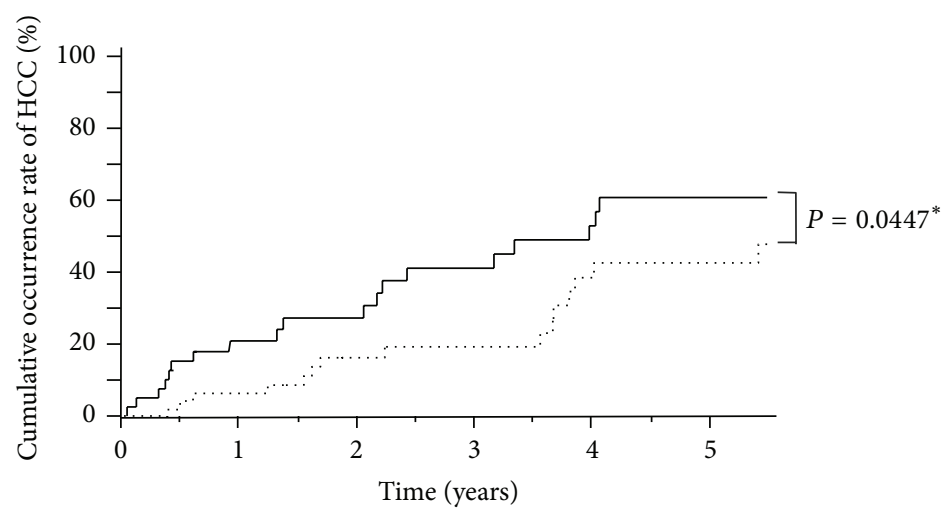

FIGURE 7: In a comparison of the cumulative occurrence rate of HCC after treatment between the BRTO group (group A) and the non-BRTO group, the log-rank test revealed that the cumulative occurrence rate of HCC after treatment was significantly higher in the BRTO group, compared with that in the non-BRTO group $\left(P=0.0447^{*}\right) .{ }^{*} P$ values less than 0.05 were considered significant.

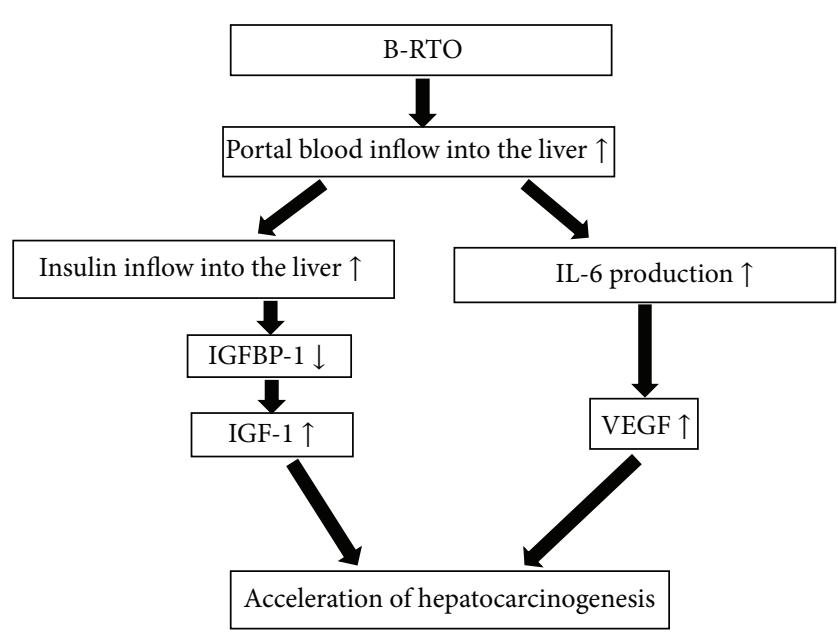

FIGURE 8: The proposed mechanisms to accelerate hepatocarcinogenesis due to B-RTO.

the growth of HCC may be accelerated by increased IL- 6 production.

First of all, increased blood flow through the portal vein in the liver with a high carcinogenic potential may have the advantage of accelerating liver regeneration and the disadvantage of contributing to liver carcinogenesis. Therefore, BRTO may be a so-called "double-edged sword" treatment. To overcome this problem, it is necessary to follow up cases after BRTO rigorously by imaging examinations of the liver, to pay close attention for early detection of HCC and to facilitate for rapid planning of comprehensive treatment.

We were confronted with some limitations in this study. First, our study is retrospective which makes it difficult to compare to other generally prospective studies. Second, there are some articles in which intrahepatic portal flow is increased after BRTO; however, we did not measure the actual portal pressure. More suggestive data, for example, the hepatic venous pressure gradient (HVPG) or the portal flow estimated by Doppler ultrasound, should be compared with pre- and post-BRTO.

\section{Conclusions}

This study is the first report to investigate the incidence of HCC after BRTO. We have suggested the potential for BRTO to accelerate HCC occurrence by increasing portal blood flow. However, there is no objection to BRTO as an important therapy for improving the rupture of gastric varices and hepatic encephalopathy. After BRTO, we must keep in mind the likelihood of HCC progression in the short period, and it is important to make an effort for careful observation and early detection of HCC. The weakness of this study lies in it being a retrospective study; thus, it is necessary to clarify the relationship between HCC and BRTO by the accumulation of new cases and a prospective study in the future.

\section{Conflict of Interests}

The authors declare that there is no conflict of interests regarding the publication of this paper.

\section{Acknowledgments}

The authors gratefully thank Professor Kengo Yoshimitsu, Chair of Department of Radiology, Fukuoka University Faculty of Medicine, and Associate Professor Hideyuki Higashihara, Department of Radiology, Chikushi Hospital, Fukuoka University, for performing a large number of BRTO procedures. And they also thank Ms. Atsuko Ishibashi for her assistance in data collection.

\section{References}

[1] H. Kanagawa, S. Mima, H. Kouyama, K. Gotoh, T. Uchida, and K. Okuda, "Treatment of gastric fundal varices by balloonoccluded retrograde transvenous obliteration," Journal of Gastroenterology and Hepatology, vol. 11, no. 1, pp. 51-58, 1996.

[2] T. Kim, H. Shijo, H. Kokawa et al., "Risk factors for hemorrhage from gastric fundal varices," Hepatology, vol. 25, no. 2, pp. 307312, 1997.

[3] H. J. Grossman, V. L. Grossman, and P. S. Bhathal, "The effect of hepatocyte enlargement on the hemodynamic characteristics of 
the isolated perfused rat liver preparation," Hepatology, vol. 27, no. 2, pp. 446-451, 1998.

[4] P. S. Bhathal and H. J. Grossman, "Reduction of the increased portal vascular resistance of the isolated perfused cirrhotic rat liver by vasodilators," Journal of Hepatology, vol. 1, no. 4, pp. 325-337, 1985.

[5] H. Tanaka, K. Hirohashi, S. Kubo, T. Shuto, I. Higaki, and H. Kinoshita, "Preoperative portal vein embolization improves prognosis after right hepatectomy for hepatocellular carcinoma in patients with impaired hepatic function," British Journal of Surgery, vol. 87, no. 7, pp. 879-882, 2000.

[6] T. Fukuda, S. Hirota, and K. Sugimura, "Long-term results of balloon-occluded retrograde transvenous obliteration for the treatment of gastric varices and hepatic encephalopathy," Journal of Vascular and Interventional Radiology, vol. 12, no. 3, pp. 327-336, 2001.

[7] A. Vallet-Pichard, V. Mallet, B. Nalpas et al., "FIB-4: an inexpensive and accurate marker of fibrosis in HCV infection. Comparison with liver biopsy and fibrotest," Hepatology, vol. 46, no. 1, pp. 32-36, 2007.

[8] H. Yoshida, Y. Shiratori, M. Moriyama et al., "Interferon therapy reduces the risk for hepatocellular carcinoma: national surveillance program of cirrhotic and noncirrhotic patients with chronic hepatitis C in Japan," Annals of Internal Medicine, vol. 131, no. 3, pp. 174-181, 1999.

[9] K. Ikeda, M. Kobayashi, Y. Kawamura et al., "Stage progression of small hepatocellular carcinoma after radical therapy: comparisons of radiofrequency ablation and surgery using the Markov model," Liver International, vol. 31, no. 5, pp. 692-699, 2011.

[10] T. Hori, K. Nagata, S. Hasuike et al., "Risk factors for the local recurrence of hepatocellular carcinoma after a single session of percutaneous radiofrequency ablation," Journal of Gastroenterology, vol. 38, no. 10, pp. 977-981, 2003.

[11] Y. Komorizono, M. Oketani, K. Sako et al., "Risk factors for local recurrence of small hepatocellular carcinoma tumors after a single session, single application of percutaneous radiofrequency ablation," Cancer, vol. 97, no. 5, pp. 1253-1262, 2003.

[12] K. Iwata, T. Sohda, S. Nishizawa et al., "Postoperative recurrence in hepatocellular carcinoma: comparison between percutaneous ethanol injection and radiofrequency ablation," Hepatology Research, vol. 36, no. 2, pp. 143-148, 2006.

[13] S. Sherlock, "Viruses and hepatocellular carcinoma," Gut, vol. 35, no. 6, pp. 828-832, 1994.

[14] K. Saito, S. Inoue, T. Saito et al., "Augmentation effect of postprandial hyperinsulinaemia on growth of human hepatocellular carcinoma," Gut, vol. 51, no. 1, pp. 100-104, 2002.

[15] H. B. El-Serag, T. Tran, and J. E. Everhart, "Diabetes increases the risk of chronic liver disease and hepatocellular carcinoma," Gastroenterology, vol. 126, no. 2, pp. 460-468, 2004.

[16] J. A. Davila, R. O. Morgan, Y. Shaib, K. A. McGlynn, and H. B. El-Serag, "Diabetes increases the risk of hepatocellular carcinoma in the United States: a population based case control study," Gut, vol. 54, no. 4, pp. 533-539, 2005.

[17] A. A. Samani, S. Yakar, D. LeRoith, and P. Brodt, "The role of the IGF system in cancer growth and metastasis: overview and recent insights," Endocrine Reviews, vol. 28, no. 1, pp. 20-47, 2007.

[18] M. Makuuchi, B. L. Thai, K. Takayasu et al., "Preoperative portal embolization to increase safety of major hepatectomy for hilar bile duct carcinoma: a preliminary report," Surgery, vol. 107, no. 5, pp. 521-527, 1990.
[19] S. Kobayashi, M. Nagino, Y. Yokoyama, Y. Nimura, and M. Sokabe, "Evaluation of hepatic interleukin-6 secretion following portal vein ligation using a minimal surgical stress model," Journal of Surgical Research, vol. 135, no. 1, pp. 27-33, 2006.

[20] W. E. Naugler and M. Karin, "The wolf in sheep's clothing: the role of interleukin-6 in immunity, inflammation and cancer," Trends in Molecular Medicine, vol. 14, no. 3, pp. 109-119, 2008.

[21] M. F. Neurath and S. Finotto, "IL-6 signaling in autoimmunity, chronic inflammation and inflammation-associated cancer," Cytokine and Growth Factor Reviews, vol. 22, no. 2, pp. 83-89, 2011.

[22] H. Nakagawa, S. Maeda, H. Yoshida et al., "Serum IL-6 levels and the risk for hepatocarcinogenesis in chronic hepatitis C patients: an analysis based on gender differences," International Journal of Cancer, vol. 125, no. 10, pp. 2264-2269, 2009. 


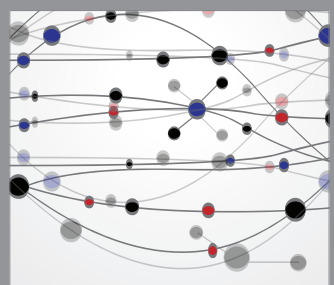

The Scientific World Journal
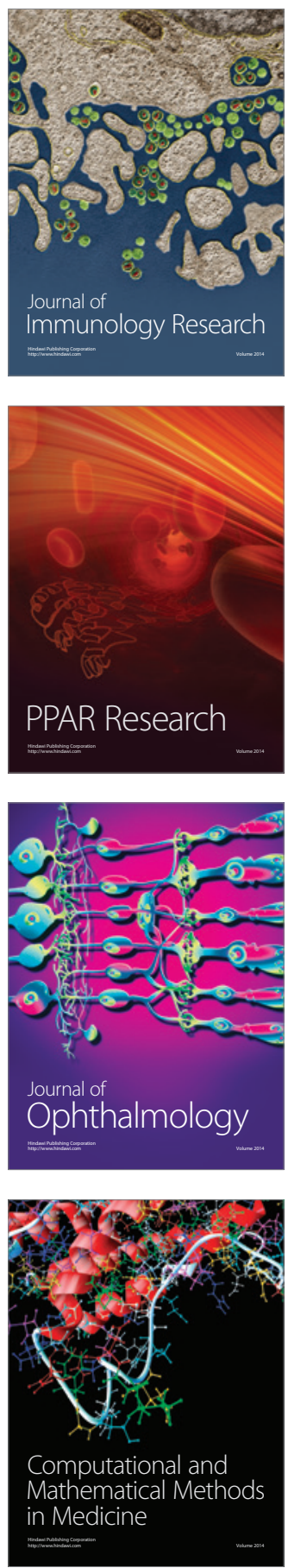

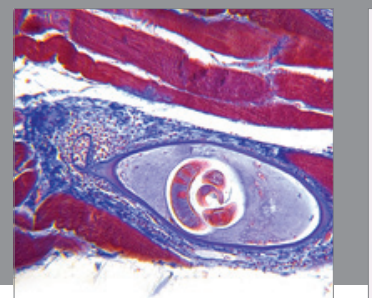

Gastroenterology

Research and Practice
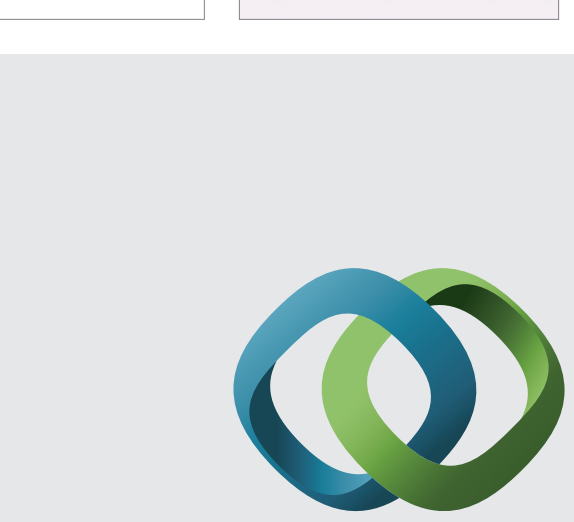

\section{Hindawi}

Submit your manuscripts at

http://www.hindawi.com
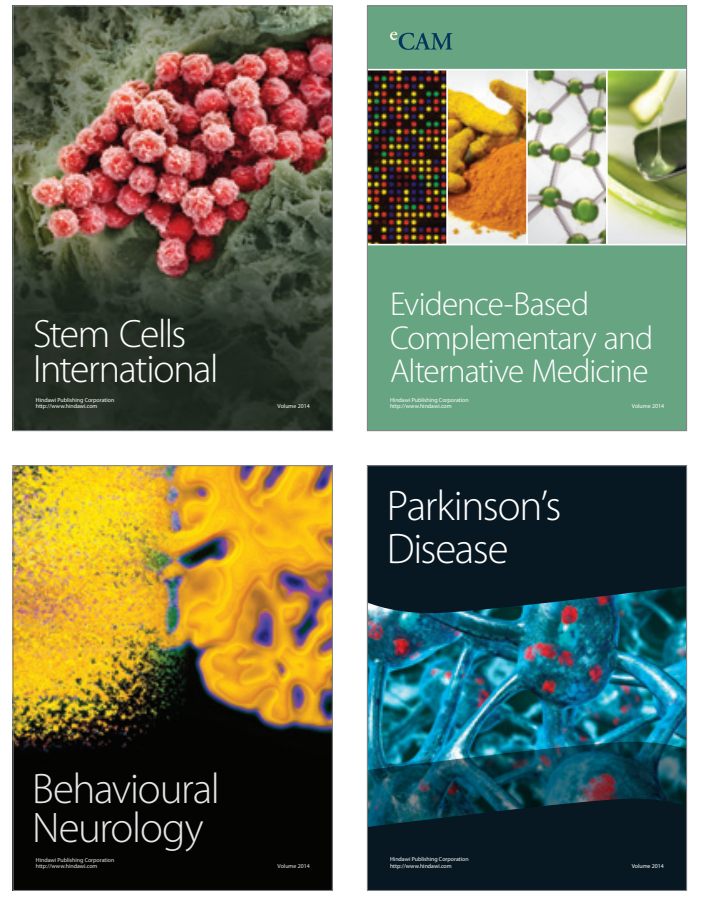
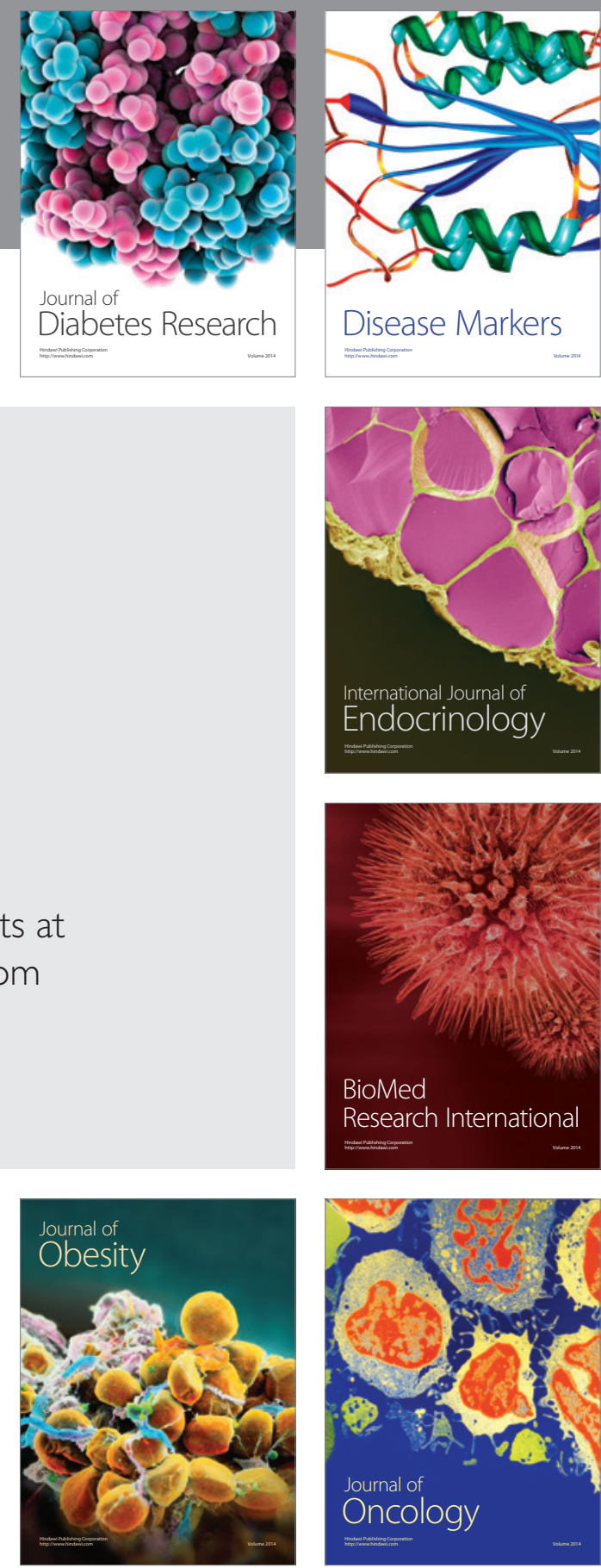

Disease Markers
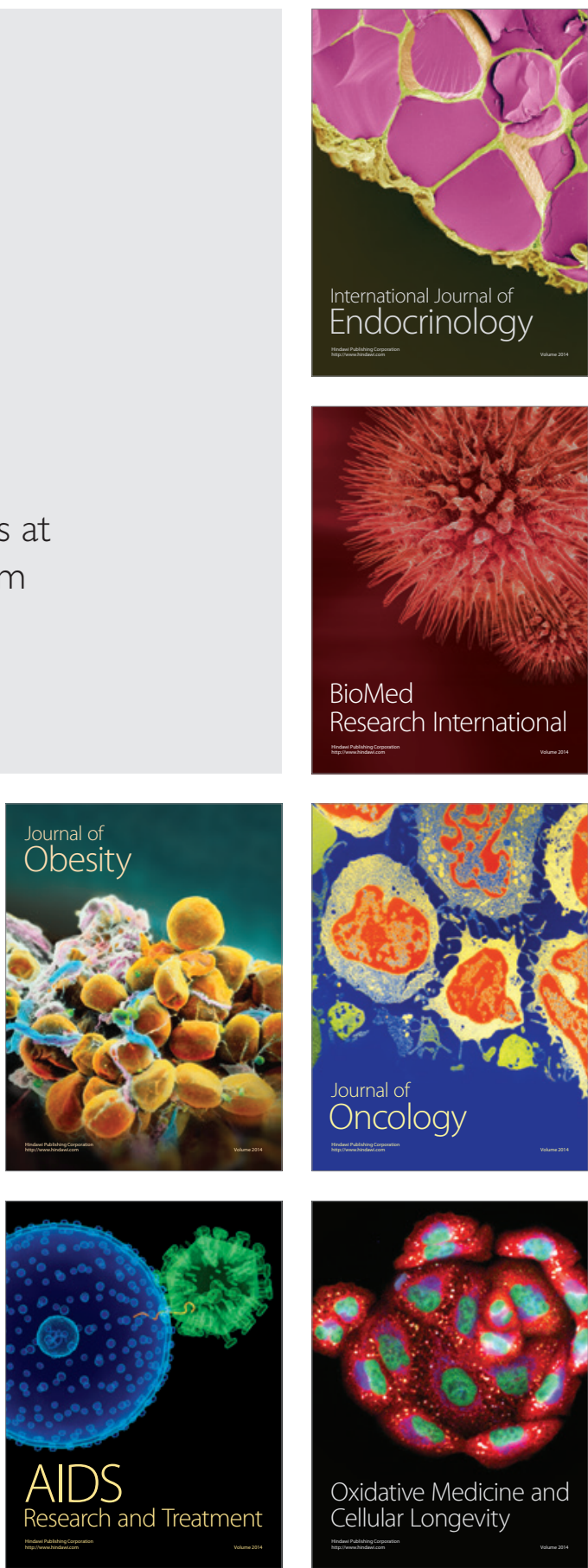